\title{
Synthesis and Characterization of Bioactive Amphiphilic Graft Copolymers with Hydrophilic Poly (1, 3-dioxolane) Side Chains
}

\author{
BOUKAR MOHAMMED ${ }^{1,2}$, SALMI HASSANIA ${ }^{1}$ and OULD KADA SEIGHIR
}
1Department of Sciences, Faculty of Sciences and Technology, University of Bechar 08000, Algeria.
${ }^{2}$ Laboratory of Macromolecule Chemical- Physical, University of Oran,
Department of Sciences, El Menouar Bp 1524 Oran 31000. Algeria.
${ }^{*}$ Corresponding author E-mail: mohammedmebarek@yahoo.fr

http://dx.doi.org/10.13005/ojc/300119

(Received: December 30, 2013; Accepted: February 06, 2014)

\begin{abstract}
2-oxypropylmethacrylat termined poly (1, 3 dioxolane) macromonomers having various chain lengths, were prepared by cationic polymerization using $\mathrm{H}_{2} \mathrm{SO}_{4}$ as initiator. The end group is introduced via the termination (end capping) using 2-hydroxypropylmethacrylate (2-HPMA). The amphiphilic graft copolymer is formed by the hydrophilic poly (1,3- dioxolane) side chain and hydrophobic poly 2-hyèdroxypropylmethacrylate backbone with various chain lengths, were synthesized by free radical copolymerization of poly (1, 3 dioxolane) $\omega$ - oxypropylmethacrylate in the presence of 2 hydroxypropylmethacrylate using benzyle peroxide as initiator.
\end{abstract}

Key words: Cationic polymerization; macromonomer; radical free copolymerization; Amphiphatic, Graft copolymers, Surface tension, poly (1, 3 dioxolane).

\section{INTRODUCTION}

The graft copolymers have received attention recently ${ }^{1-3}$, they have many important application ${ }^{4-10}$ in the polymer industry, mainly as surface modifiers for uses as coating, adhesives and dispersants; as compatbilizing agents in polymers blends, but also biomedical use. The amphiphilic graft copolymers are investigated widely because of their special physical and chemical properties and self assembly morphologies ${ }^{11}$ in order to obtain amphiphilic graft copolymers with hydrophilic side chains of defined size, we have chosen a method using a precursory polymer ${ }^{12-17}$.

\section{EXPERIMENTAL}

\section{Materials}

The monomers 1, 3 dioxolane (DXL prolabo) were purified by vacuum distillation, sulfuric acid $\left(\mathrm{H}_{2} \mathrm{SO}_{4}\right.$, prolabo). The free radical initiator employed for the polymerization was benzyl peroxide 
(Fluka) all solvents chloroform, dicloromethan, tetrahydrofurane and cyclohyxane, were purified by standard procedures.

\section{Measurements}

UV spectra were record on a Spectronic Gensys 5 spectrometry using chloroform as a solvent. FT, IR spectra were performed on a nocolet 520 by $4000-400 \mathrm{~cm}^{-1}$ range spectrophotometer, the $1 \mathrm{H}-\mathrm{NMR}$ and 13C NMR spectra of the polymers were recorded using Brucker DPX $300 \mathrm{MHZ}$ spectrometer at room temperature, tetramethylsilane (TMS) is the internal standard. Viscosities of the polymer solutions $(0,1-1 \mathrm{~g} / \mathrm{dl}$ were measured with a capillary viscosimeter (ubbelhode viscosimeter).
Synthesis of poly (1, 3- dioxolan) macromonomers

1, 3- dioxolane is dissolved in $10 \mathrm{ml}$ of freshly distilled chloroforme and placed under nitrogen atmosphere in a three- necked flask equipped with a condenser and magnetic stirring. The 2-hydoxypropylmehacrylate is added to the reaction mixture, the polymerization is terminated by introducing dimethylamine. The precipate is filtered off, washed with ether and dried in vacuum. The synthesis of a macromonomer of 1, 3-dioxolane is shown in scheme below.<smiles>CC(O)COCCOCCOCC(C)C(=O)OCC(C)COC(=O)C(C)C</smiles>

Table 1 list the experiment conditions of the reaction

Table 1: Experimental conditions and yields of cationic polymerization of 1, 3- dioxolane initiated by $\mathrm{H}_{2} \mathrm{SO}_{4}$

\begin{tabular}{lccccc}
\hline Product & $\begin{array}{c}{[\mathrm{DXL}]} \\
(\mathbf{m o l} / \mathbf{l})\end{array}$ & $\begin{array}{c}{[\mathbf{2 H P M A}]} \\
(\mathbf{m o l} / \mathbf{l})\end{array}$ & $\begin{array}{c}\left.\mathbf{H}_{\mathbf{2}} \mathbf{S O}_{\mathbf{4}}\right] \\
(\mathbf{m o l} / \mathbf{l})\end{array}$ & $\begin{array}{c}\text { [DXL]/ } \\
{[\mathbf{2 H P M A}]}\end{array}$ & Rendement \\
\hline $\mathrm{BMD}_{1}$ & 12,550 & 0,658 & 0.025 & 19,07 & $86 \%$ \\
$\mathrm{BMD}_{2}$ & 13,127 & 0,327 & 0.688 & 40,14 & $73 \%$ \\
$\mathrm{BMD}_{3}$ & 12,550 & 0,344 & 0,125 & 36,48 & $78 \%$ \\
$\mathrm{BMD}_{4}$ & 12,550 & 0,172 & 0,3546 & 72,96 & $82 \%$ \\
$\mathrm{BMD}_{5}$ & 13,127 & 0,1635 & 0,456 & 80,28 & $88 \%$ \\
\hline
\end{tabular}

Preparation of the 1, 3- dioxolan graft copolymer

The polymerization is initiated by benzoyl peroxide. After 24 hours, the copolymer were precipitated into excess of cyclohexane in order to eliminate the poly (methylmethacrylate) homopolymer, dried under vacuum and dissolved in chloroform and precipitated again in water for eliminating the 1, 3dioxolan monomer. The experimental condition is reported in Table 2.

\section{RESULTS AND DISCUSSION}

UV analysis of oxypropylmethacryloyle end chain

UV analysis is used; on one band $(\lambda=220$ $\mathrm{nm}$ ) to observe the fixation of chromophoric group oxypropylmethacrylate on the 1,3-dioxolane end chain on the other hand to calculate number- average molecular weight $\mathrm{Mn}$ of the macromonomer. 
Table 2: The experimental conditions of formation the copolymer 1, 3-dioxolane- g- 2- hydrpropylmethacrylate

\begin{tabular}{lccc}
\hline$[\mathrm{P}]_{0}$ Mole/l & {$[\mathrm{M}]_{0}$ mole/ I } & {$[\mathrm{PB}]$ mole/l } & {$[\mathrm{M}]_{0} /[\mathrm{P}]_{0}$} \\
\hline 0,124 & 0,07 & $1,3.10^{-1}$ & 9,66 \\
0,124 & 0,401 & $0,061.10^{-1}$ & 3,23 \\
\hline Notes: [P]0: initial concentration of poly (1, 3- dioxolane) macromonomer calculated \\
by UV analysis; [M]0: initial molar concentration of 2hydrxypropylmethacrylate \\
comonomer; [PB]0: initial molar concentration on benzoyl peroxide initiator; \\
Poly(DXL-g- 2HPMA); poly (1, 3-dioxolan graft poly methylmethacrylat), P(DXL- g- \\
2-HPMA): poly 1, 3 dioxolane-g- 2-hydroxypropylmethacrylate.
\end{tabular}

Table 3: Numbers of average molecular weights of 1, 3-dioxolane macromonomers calculate from UV analysis $(\varepsilon=8643, \lambda=220 \mathrm{~nm}$, solvent: dichloromethane $)^{17}$

\begin{tabular}{lcc}
\hline Product & $\boldsymbol{M n}_{\text {théorical }}$ & $\boldsymbol{M} \boldsymbol{n}_{\mathbf{u v}}$ \\
\hline $\mathrm{M}_{1,}$ & 1500 & 1500 \\
$\mathrm{M}_{2}$ & 2000 & 2000 \\
$\mathrm{M}_{3}$ & 2500 & 2500 \\
$\mathrm{M}_{4}$ & 3000 & 3000 \\
\hline
\end{tabular}

By assuming, that only one of the chromophoric groups is attached to end polymeric chain and by using molecular extinction coefficient of molecular model, in this case, the 2-hydrxypropylmethacrylate (2-HPMA), we can calculate the molecular weight of our samples.

Thus, starting from a calibration curve representing the variation of the optical density
Table 4: Number average molecular weight Mn of 1, 3- dioxolane macromonomers determined by viscosity $\left(K=2,310^{-2}\right.$, $\alpha=0,65, \mathbf{T}=25^{\circ} \mathrm{C}$ solvent: dicloromethane)

\begin{tabular}{lc}
\hline Macromonomer & Mn(viscosity) \\
\hline$M_{1}$ & 1600 \\
$M_{2}$ & 2100
\end{tabular}

according to the concentration of 2-HPMA in chloroforme. We have obtained a curve which follows BEER LMABERT's low, which can calculate the molecular extinction coefficient of 2-HPMA taken as model $(\varepsilon=8463$ at $\lambda=220 \mathrm{~nm}$ ) in chloroforme.

The number average molecular weights calculated from UV analysis are listed in Table 3.

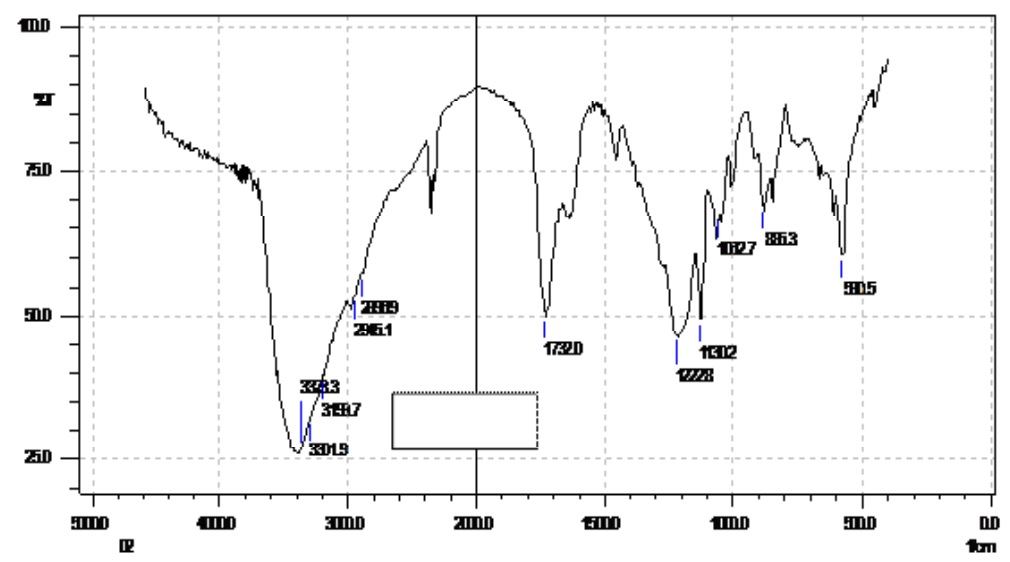

Fig. 1: FT- IR of the $\omega$-oxypropylmethacrylate 1,3 dioxolane in chloroforme 


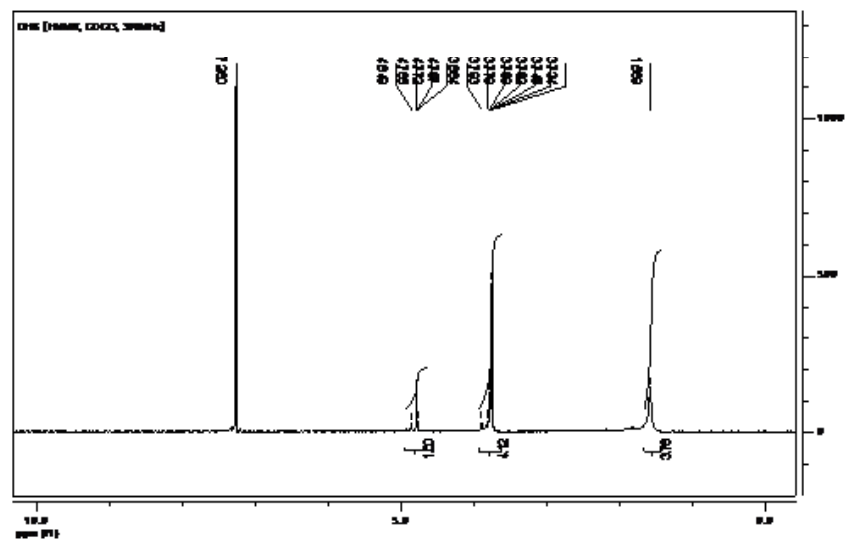

Fig. 2: ${ }^{1} \mathrm{H}$ - NMR spectrum of the $\omega$-oxypropylmethacrylate 1,3 dioxolane in $\mathrm{CDCl}_{3}$

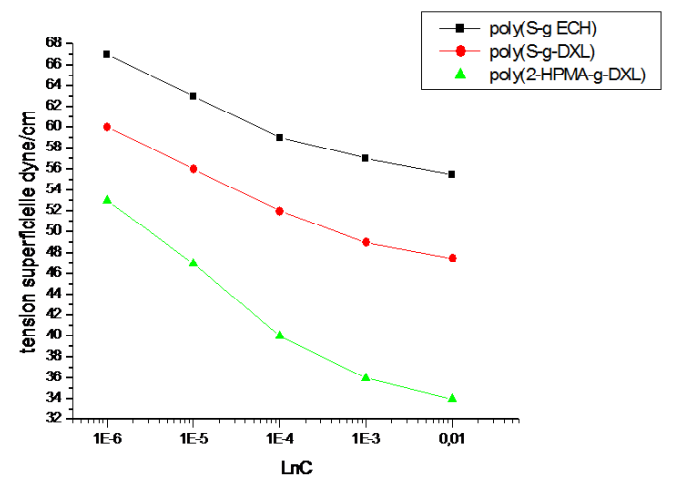

Fig. 3: The logarithmic plot of superficial tension of the graft copolymer solution vs, concentration

Viscosity measurements used to obtain the average molecular weights of polymers produced the Mark- Houwink equation was used to calculate the viscosity average molecular weight ${ }^{18-19}$. The values of $\mathrm{Mn}$ are presented in Table 4.

We notice that the values of the numberaverage molecular weight determined by UV analysis and by viscosimetry are relatively in accordance with those calculated theoretically. This results suggest that the contribution of transfer reactions could by much less important, which could mean that the reaction occurs, according to a "living form".

\section{Analysis FT-IR}

The FT-IR spectra of 1, 3-dioxolane macromonomers (Fig. 1) shows the most characteristic absorption at $1732 \mathrm{~cm}^{-1}$ corresponds to the carbonyl group, the peak at $2945 \mathrm{~cm}^{-1}$ due to the stretching vibration of methyl group in the end of chain, the peak at $1222 \mathrm{~cm}^{-1}$ is attributed to stretching vibration band of the double bond. The bands at 1082 $\mathrm{cm}^{-1}$ are assigned to ester faction. The peak at 886 $\mathrm{cm}^{-1}$ and $580 \mathrm{~cm}^{-1}$ corespond to bonding vibration, we notice the disappearance of characteristic band at $1630 \mathrm{~cm}^{-1}$ and $981 \mathrm{~cm}^{-1}$ related to the double bond of the monomer and a new characteristic peak appears at $1384 \mathrm{~cm}^{-1}$ due to the oxypropylmethacrylate double bond.

The ${ }^{1} \mathrm{H}-\mathrm{NMR}$ spectra of 1 , 3- dioxolane macromonomer was record in $\mathrm{CDCl}_{3}$ solution using a DPX 300 Brucker spectrometer; the ${ }^{1} \mathrm{H}-\mathrm{NMR}$ spectra of macromonomer is shown in Figure 2.

\section{Characterization of copolymer by ${ }^{13} \mathrm{C}-\mathrm{RMN}$}

The poy (1, 3- dioxolan)-g-PMMA was characterized by ${ }^{13} \mathrm{C}-\mathrm{RMN},{ }^{13} \mathrm{C}-\mathrm{RMN}$ analysis of poly (1, 3-dioxolan)-g-2HPMA confirms that the chain are copped with poly (2-HPMA) backbone. The ${ }^{13} \mathrm{C}-\mathrm{RMN}$ spectrum of the copolymer shows the signal with chemical shifts of about 38-42 ppm, 36- 30 ppm 
correspond to the $-\mathrm{CH}_{2}$ - carbon atms and those observed at 170-178 ppm and 57 ppm are attributed respectively to carbonyl carbon and $\mathrm{CH}$ - carbon of oxypropylmethacrylate.

\section{Determination of the surface tension of the copolymer poly (1, 3-dioxolan)-g-PMMA}

Interestingly, the amphiphilic character of the poly (1,3-dioxolan) in the graft copolymer, has been evidenced by the superficial tension measurements.

Purposely; solution of poly(1, 3-dioxolan)g-PMMA, in water, Fig. 3 shows the logarithmic plot of superficial tension of the graft copolymer solution vs, concentration (expressed in $\mathrm{g} / \mathrm{l}$ at $25^{\circ} \mathrm{C}$, the superficial tension goes down from $63 ; 3 \mathrm{~N} / \mathrm{m}$ to $53,9 \mathrm{~N} / \mathrm{m}$, demonstrating the tensioactive proprieties of the new type of amphiphilic graft copolymer.
The critical micelle concentration (CMC) has been determined from the intersection between the tangents drawn from higher concentration portion of the sigmoid plot and calculated as $10^{-4} \mathrm{~g} / \mathrm{l}$.

\section{CONCLUSIONS}

The graft copolymer of poly (1, 3dioxolan)g-MAA were prepared by radical copolymerization. Amphiphilic graft copolymers containing hydrophobic poly (2-hydroxypropylmethacrylate) backbone and hydrophilic poly (1, 3-dioxolan) side chain were synthesized by free radical copolymerization, using benzoylperoxide as initiator. The analysis by ${ }^{1} \mathrm{H}$ NMR, ${ }^{13} \mathrm{C}$ NMR, FTir and UV confirmed the sequence copolymer of poly (1,3- dioxolane)-g-P2HPMA. These materials have potential applications in various fields and in particular with the aim of pharmacy and medicine.

\section{REFERENCES}

1. Tsukahara. Y; Mizuno. K, Segawa. A, Yamashata? $Y$ study on the radical polymerization behavior of macromonomers. Macromolecules, 22: 1546- 1552 (1989).

2. Neugebauer. D, zhang. Y, pakulaaaaa; T, Sheiko. S Matyjaszewky. K densly-grafted and double grafted POE burushers via ATPR ; a route to soft elastomers. Macromolecules. 36: 6746-6755 (2003).

3. Debuigne. A, Caille. J. R, Willet. N, Jerome. $R$, synthesis of poly vinyl alcohol) containing block copolymers by combination of cobalt mediated radical polymerization and ATRP, macromolecules, 38: 9488-9496 (2005).

4. Mozali. M, Lacoste. J, Abadie. M. J. M, Synthese de copolymers styrene/isoprene/ styrene $\alpha, \omega$ - diepoxydes ; propriétés adésives. Eur, Polym, J, 28: 1241-1246 (1992).

5. Eguiburu. J. L, Berridi. M. J. F, Roman.J. S, graft copolymers for biomedical application prepared by free radical polymerization of poly(lactide) macromonomers with vinyl and acrylic monomers, Polymer, 37: 3615- 3622 (1996).

6. Soykan. C, Koskun. M, Ahmadazade. M, Ozdemi. E, phenacylmethacrylate and $\mathrm{N}$ - vinyl-2-pyrrolodone copolymers, shyntesis, characterization reactivity ratios. Pure and appl. Chem., A 37(9): 1089-1101 (2000).

7. Coulembier. O, Degree. P, barbaud. C, Urén. $\mathrm{P}$, Dubois. $\mathrm{P}$, new amphiphilic graft copolymer based on poly( $\beta$ - malic acid ; synthesis and characterization, polymer Bulletin, 52 (2004).

8. Gao. Y, Li, S, Li. H, Wang. X, synthesis of syndiotactic polystyrene-graft poly(methacrylat and poly(styrene) and syndiotactic polystyrene- graft acatic poly(styrene) by atom transfert radical polymerization, Eurp. Poly. J, 41: 2329-2334 (2005).

9. Li. Z, Li. P, Huang. J. synthesis and characterization of amphiphilic graft copolymer poly ethylene oxide)- graft- poly methacrylat) polymer, 47: $5791-5798$ (2006).

10. Chun. Y, Chlu. Y, Jie Y, Shio. W. K, Hsien. X. C, Feng. C. C ; complicated phase behavior.

11. Riess. G, micellization of block copolymers, Prog. Poly, sci., 28: 1107- 1170 (2003).

12. Ranucci. E, Spagnoli. G, Sartore. L, Bigntti. F, Ferruti. P, Schiavon. O. synthesis and molecular weight charatcterization of endfunctionalization of $\mathrm{N}$ - vinyl-2- pyrolidone 
oligomers. Macromol. Chem. Phys, 196: 763774 (1995).

13. Raccini. E, Tarabic. M, Alerston. A. new ester and lactone end functionalized N-vinylpyrolidone oligomers. Macromol. Chem. Phys, 201: 1219-1225 (2000).

14. Garibi. H; palepu. R, Tiddy. G. J. T; Hall. D. G, Wyne. J., J. Chem. Soc. Commun. 2: 115 (1990).

15. R. Indira, T. Tamizharuvi and V. Jaisankar, Orient J. Chem., 28(3): 1479-1487 (2012).

16. S. Zomorodbaksh, H.A. Anarki and E. Tavahodi, Orient J. Chem., 28(4): 1645-1650
(2012).

17. H. Bendaikha, G. Clisson, A. Khoukh, J. François, S. Ould Kada. Synthesis and characterization of amphiphilic graft copolymers of macromonomers of $(1,3-$ dixolane) with methacrylate and styrene. e-polymer, 09: 9- 28 (2009).

18. M. Fontanille, Y, Gnanou. Chimie et physicchimie de polymère, Ed, Paris, Dunod, Chap. 6 (2002).

19. M. Boukar, mémoire de magister, université d'Oran Es-Senia, Algerie (2000). 